\title{
Puente adhesivo en paciente con labio leporino y fisura velopalatina. Una solución temporal con compromiso estético
}

\author{
Adhesive bridge in a patient with cleft lip and palate. A temporary \\ solution with esthetic commitment
}

\author{
Leiva $\mathrm{N}^{1}$, Corsini $\mathrm{R}^{2}$, Nieto $\mathrm{E}^{3}$
}

\begin{abstract}
RESUMEN
En la actualidad el aumento de la demanda estética y la necesidad de procedimientos mínimamente invasivos ha llevado a un aumento de la técnica adhesiva. Ribbond® es una marca de fibra de polietileno que permite un eficiente traspaso de fuerzas, es virtualmente plegable y se adapta fácilmente a la morfología dentaria y al contorno del arco dentario. Dentro de sus principales características podemos encontrar: Biocompatibilidad, inerte, translúcido y durable. Dentro de la odontología se le han dado distintos usos para esta fibra, ya sea como mantenedor de espacio, puente adhesivo, poste y núcleo endodóntico, ferulización, restauraciones tipo inlay y estabilización postortodóncica. En el caso de los pacientes fisurados se ha hecho relevante el uso de Ribbond® como puente adhesivo ya que permite mejorar la estética y rehabilitar temporalmente los espacios desdentados causados por la ausencia de un incisivo, principalmente una vez terminado el tratamiento de ortodoncia y en espera de una rehabilitación definitiva, aún más cuando el paciente no ha terminado su crecimiento y desarrollo. En este artículo se presenta un caso clínico de un paciente con labio leporino y fisura velopalatina operado demostrativo de la técnica de rehabilitación con Ribbond®.
\end{abstract}

Rev. Clin. Periodoncia Implantol. Rehabil. Oral Vol. 6(1); 33-37, 2013.

Palabras clave: Ribbond®, fisura labiopalatina, rehabilitación estética.

\section{ABSTRACT}

Today the increasing demand of aesthetic and minimally invasive procedures has led to a boom of the adhesive technique. Ribbond $₫$ is a polyethylene fiber that allows an efficient transfer of forces, is virtually foldable and is easily adapted to tooth morphology and dental arch contour. Among its main features we can find: Biocompatibility, inert, translucent and durable. In dentistry, different uses have been given to this fiber, whether as space maintainer, adhesive bridge, endodontic post and core, splinting, inlay type restorations and postorthodontic stabilization. In the case of cleft lip and palate patients the use of Ribbond $\otimes$ as an adhesive bridge has become relevant, as it improves aesthetics and temporarily rehabilitates the edentulous spaces caused by the absence of an incisor. It is used especially after completion of the orthodontic treatment and waiting for a final rehabilitation, even when the patient has not completed their growth and development. This article presents a clinical case of a patient with lip and cleft palate operated with the rehabilitation technique using Ribbond®.

Rev. Clin. Periodoncia Implantol. Rehabil. Oral Vol. 6(1); 33-37, 2013.

Key words: Ribbond $\circledast$, cleft lip and palate, esthetic rehabilitation.

\section{INTRODUCCIÓN}

El aumento de la demanda por mantener el tejido dentario, la estética y la disminución del costo por parte del paciente, provoca que los clínicos busquen materiales y técnicas que permitan acercamientos mínimamente invasivos. El uso de la técnica adhesiva y los composites reforzados con un sistema de fibras permiten responder a estas demandas $^{(1)}$.

Los pacientes portadores de fisura de labio y velopalatina presentan condiciones aún más desafiantes para la rehabilitación de las zonas edéntulas, ya que ésta no sólo debe cumplir con las condiciones de estética y funcionalidad, además debe ser flexible y acompañar el movimiento de los segmentos óseos cuando no han sido injertados.

Es importante intervenir de la menor manera posible las piezas adyacentes a la zona edéntula y privilegiar el tratamiento conservador.

Por lo mismo, la técnica adhesiva utilizando un sistema de fibras presenta como una alternativa viable de rehabilitación provisoria para este tipo de pacientes, durante el periodo de crecimiento y en espera de la rehabilitación definitiva.

Existen diferentes tipos de fibras como: Fibras de vidrio, de carbón, de Kevlar, de Vectran y de polietileno; las cuales han sido añadidas a los materiales resinosos. Las fibras de vidrio consisten en filamentos de vidrio entrelazados, estas mejoran la resistencia al impacto de los composites. Poseen excelentes propiedades estéticas, pero no se adhieren fácilmente a la matriz resinosa. Las fibras de carbón, previenen la fractura por fatiga de material y fortalecen la estructura de los composites; pero, ellas presentan un color oscuro, lo que es indeseable estéticamente $^{(2-5)}$. Las fibras de Kevlar están compuestas por poliamidas aromáticas, son la evolución de las poliamidas de nylon. Éstas aumentan la resistencia al impacto de los materiales resinosos. Sin embargo, son antiestéticos, y en consecuencia, su uso es limitado ${ }^{(2,6)}$. Las fibras de Vectran son sintéticas de nueva generación, hechas de poliésteres aromáticos. Estas muestran una buena resistencia a la abrasión y a la fuerza de impacto, pero son costosas y de difícil manejo.

Las fibras de polietileno mejoran la resistencia al impacto, el módulo de elasticidad y la fuerza flexural de los composites. A diferencia de las fibras de carbón y de Kevlar, estas fibras son prácticamente invisibles dentro de la matriz resinosa y debido a esta razón, parecen ser las más apropiadas y estéticamente resistentes para los materiales resinosos ${ }^{(2,5)}$.

1. Directora Instituto de Rehabilitación de Malformaciones y Deformaciones Maxilofaciales, IRMADEMA. Facultad de Odontología, Universidad de Chile. Chile.

2. Estadía en IRMADEMA 2011-2012. Chile.

3. Prof. Titular. Universidad Mayor. Rehabilitador Oral. Chile.

Correspondencia autor: Noemí Leiva. irmadema@odontologia.uchile.cl. Trabajo recibido el 30/04/2012. Aprobado para su publicación el $04 / 07 / 2012$. 


\section{MARCO TEÓRICO}

Ribbond $\circledast$ es una fibra hecha de polietileno de ultra alto peso molecular, que tiene sus inicios desde $1992^{(2)}$. Es tratado con plasma de gas frío para aumentar la adhesión a los materiales sintéticos de restauración, la red de fibras especiales de este material permite un eficiente traspaso de fuerzas, es virtualmente plegable y debido a esto se adapta fácilmente a la morfología dentaria y al contorno del arco dentario.

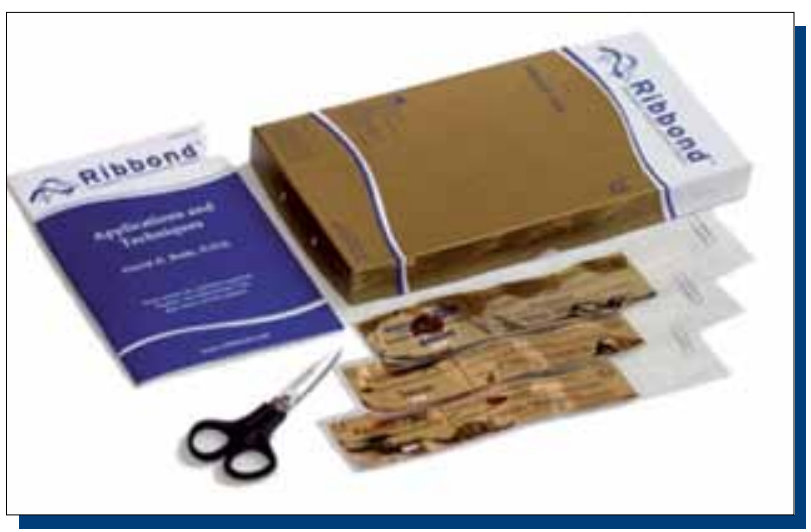

Figura 1. Set completo con tijera accesoria.

Al ser un material traslúcido es altamente estético y esta cualidad también permite que la fotopolimerización de los composites usados en la rehabilitación con Ribbond® pueda ser realizada sin alteraciones.

Estas fibras pueden ser cortadas usando una herramienta adecuada (tijera microdentada) $\sin$ dañarlas ${ }^{(1,7,8)}$

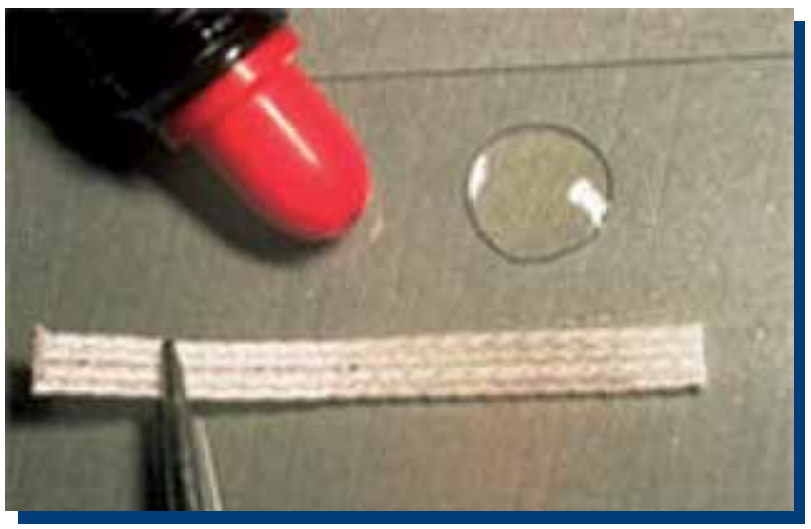

Figura 2. Cinta de Ribbond de $2 \mathrm{~mm}$.

El Ribbond $\circledast$ es un conjunto de 215 fibras, la que posee un muy alto coeficiente de elasticidad (117 GPa); esto significa que tendrá resistencia al estiramiento y torsión.

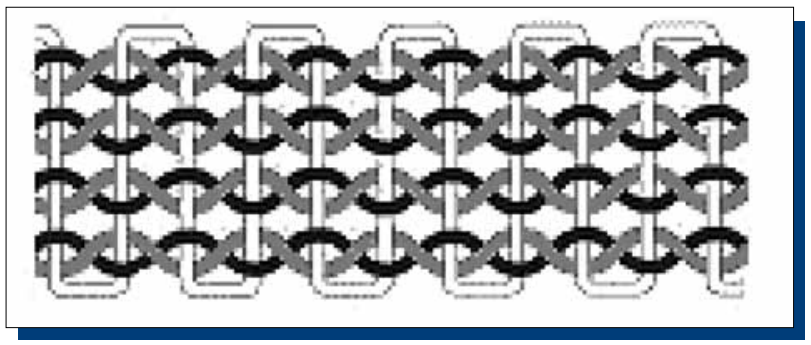

Figura 3. Tejido de cadenas entrelazadas.

También tienen una muy alta resistencia a la tracción (3 GPa) como resultado de su configuración de "puntada corta" y su buena adaptabilidad. Estas fibras de Ribbond $®$ también se caracterizan por su resistencia al impacto, la cual es 5 veces mayor que el hierro. Al ser translúcidas adquieren el color de la resina en la cual son añadidas. Las fibras de Ribbond $\AA$ absorben agua rápidamente debido al tratamiento de "plasma gaseoso" al que son expuestas ${ }^{(1)}$. Debido a este tratamiento es que las fibras no pueden quedar expuestas al medio bucal, por lo tanto deben ser manipuladas bajo aislación estricta, no puede haber contacto directo con las manos o con otros elementos que le proporcionen contaminantes, para lo cual el fabricante proporciona guantes de algodón, pero aconseja el mínimo contacto antes de su utilización, ya que la contaminación disminuye la adhesión.

El uso de composite reforzado con fibras (CRF) provee un notable incremento en la resistencia a las fuerzas flexural a la estructura completa y permite que la vida de estas CRF restauraciones aumente ${ }^{(1,7)}$

Las fibras de polietileno mejoran la fuerza de impacto, el módulo de elasticidad y la fuerza flexural de los materiales de composite, lo que ayuda a prevenir la fractura debido al alto stress asociado a la masticación (Valittu PK)(9). Las fibras de polietileno son casi invisibles en una matriz resinosa ${ }^{(1)}$.

De acuerdo a la literatura, la fuerza mecánica entregada por el refuerzo de fibras es generada por la capacidad del material de disipar las líneas de tensión y microfisuras internas que podrían causar fracturas catastróficas en un material más rígido ${ }^{(1,4)}$.

El hecho que las fibras no estén posicionadas longitudinalmente y en cambio estén trenzadas alternando patrones, aparentaría incrementar la dispersión de las líneas de tensión internas y por consiguiente, provee resistencia a la fractura. El uso de fibras de colores neutrales provee una estética mejorada y permite la difusión de la luz natural a través de cuerpo de la resina ${ }^{(1)}$.

Dentro de sus principales características podemos encontrar: biocompatibilidad (a diferencia de la fibra de vidrio, si en algún momento la Ribbond $®$ se corta con un instrumento rotatorio, las partículas resultantes y las fibras expuestas no serán un riesgo de biocompatibilidad con el paciente), inerte, translúcido, durabilidad (es la fibra de refuerzo más durable disponible en la industria dental). El tejido de cadenas de fibras interconectadas provee una estabilidad multi-direccional por la transferencia de tensión a través de toda la red de fibras. Esto previene fracturas en la resina. Permite ser almacenado a temperatura ambiente. Además, se presenta en variados grosores $(2,3$ y $4 \mathrm{~mm}$ ) y presenta una estética adecuada.

Ribbond ${ }^{\circledR}$ se adhiere a composites y resinas acrílicas. Por medio de los procedimientos estándar, recomendados por cada fabricante según la marca comercial que se esté usando, la resina o composite adhiere a: esmalte, dentina, metal, porcelana, composite. Es flexible, prácticamente no tiene memoria, no se desenlaza (desarma) al cortarse, ni se expande al adaptarse.

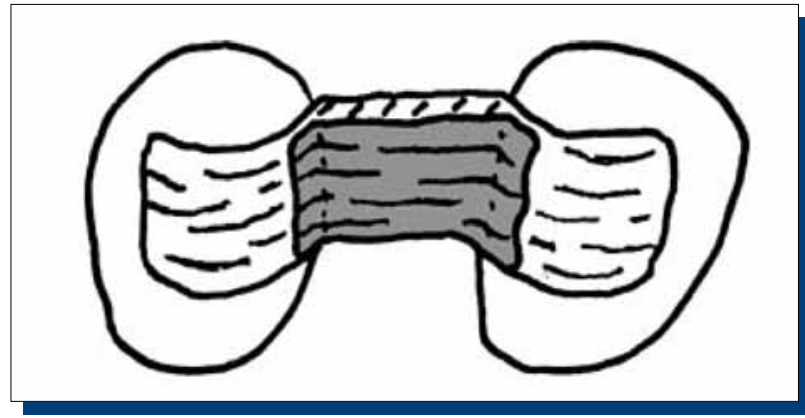

Figura 4. Una forma de colocación de la cinta.

Dentro de la odontología existen aplicaciones diferentes para las fibras de polietileno tipo Ribbond $((10)$.

- Como mantenedor de espacio, permitiendo una inserción rápida, en un procedimiento de una sola visita sin necesidad de laboratorio; la que no posee riesgo de dañar el diente pilar y de fácil aseo $^{(2,11,12)}$.

- Como puente adhesivo(2), a pesar de que una pieza dentaria anterior posee una funcionalidad mecánica, es el compromiso de la estética facial asociada a la pérdida de un diente la preocupación primordial del paciente. El reemplazo estético inmediato del diente perdido será requerido. Este reemplazo puede ser temporal o semitemporal.

- Cuando la restauración no requiere de preparación dentaria, la remoción de la estructura dentaria está contraindicada para la colocación de una restauración provisoria. Las prótesis adhesivas pueden ser tan simples como un puente de una pieza dentaria o una dentadura parcial unida por medio de resina reforzada ${ }^{(13)}$. 
El estudio clínico retrospectivo de Piovesan et al. ${ }^{(10)}$ sugiere que una dentadura parcial realizada con resina reforzada, puede ser una alternativa funcional y estética adecuada para reemplazar un diente perdido. Unlu y Belli(15) concluyeron que las resinas reforzadas con fibras de polietileno funcionan adecuadamente durante un seguimiento clínico de 3 años. En otro reporte, una tasa de un $95 \%$ de sobrevivencia funcional fue descrita, en un periodo de seguimiento de 4.3 años.

Grandini et al. ${ }^{(2,16)}$ sugirieron que la restauración de dientes tratados endodónticamente con postes de fibra y resinas directas es una opción real de tratamiento, que a corto plazo conserva el remanente dentario y resulta en una adecuada aceptación por parte del paciente.

También, es comúnmente ocupada como ferulización después de una lesión traumática para estabilizar una subluxación, luxación, avulsión o fractura radicular de una pieza dentaria ${ }^{(2)}$.

Strassler y col. ${ }^{(17)}$ reportaron el uso de fibras de polietileno para estabilización postortodóntica, como contención, para reemplazo dentario y férula periodontal. Ellos ferulizaron 64 dientes en 30 pacientes utilizando Ribbond $\circledast$. Los resultados clínicos se basaron en 12-48 meses de evaluación. Todas las férulas periodontales y contenciones fueron exitosas y ninguna presentó descementaciones o caries residuales. En dos pacientes, a los cuales se les realizó contención maxilar, se expusieron las fibras del Ribbond® debido a la función oclusal, por esto estas zonas fueron cubiertas con resina fluida. Sólo uno de 9 dientes naturales o puentes de resina se fracturó durante el estudio. Aunque la fractura a nivel de la resina fue clara, el intermediario no se separó del diente adyacente debido a que el Ribbond® lo mantuvo en su lugar ${ }^{(1)}$.

A pesar de lo anteriormente expuesto, es importante considerar que este material puede favorecer la retención de placa, la impactación alimenticia y la enfermedad periodontal. Por lo que es crítica una higiene oral minuciosa para mantener la salud y la apariencia de los resultados obtenidos con el tratamiento(1)

Como restauraciones tipo inlay, la mayoría de los estudios reportan períodos de evaluación de corto plazo ${ }^{(18,19,20)}$, por lo cual existe limitada información disponible sobre los determinantes de su desempeño y razones de falla de los inlays de resina mejorada con fibras $^{(18,21,22)}$. De hecho, parece haber unos pocos estudios clínicos de largo plazo que reportan tasas de sobrevivencia de inlays posteriores de polietileno reforzados con fibras de entre un 55 a un $86^{(18,23,24,25)}$.

En un estudio realizado por Cenci et al. el año 2009, se reportó la tasa de sobrevivencia de las restauraciones posteriores de composite reforzado con fibras durante un periodo de seguimiento de 8 años. Se observó una sobrevivencia total de 7 años, con un porcentaje de $81.8 \%$ de las restauraciones ${ }^{(18)}$.

La durabilidad de las dentaduras parciales realizadas mediante restauraciones de composite reforzadas depende de variados factores, incluyendo el material, el arco rehabilitado, factores propios del paciente y del odontólogo. Más aún, factores del paciente así como higiene oral, hábitos de alimentación, medidas preventivas, disponibilidad de fluoruración, cumplimiento en los controles y cooperación durante el tratamiento y el ambiente oral. Todos estos son temas relevantes al momento de considerar la durabilidad de una restauración ${ }^{(18,22)}$.

La razón más importante por su preferencia es la manejabilidad de la cinta. Es importante considerar la estabilidad que da el tejido, ya que la cinta es prácticamente libre de memoria, lo que asegura la adaptación estrecha y precisa. En el caso de una férula periodontal, la colocación de las fibras estrechamente contra la superficie de los dientes hace que se adhiera como una estructura laminada.

Un compuesto reforzado con fibras se hace más fuerte cuando la fibra está recubierta y cercana a los dientes, eliminando la falta de cohesión de la resina en la fibra si no está muy adaptada a los dientes.

En el caso de puentes $u$ otras estructuras de sobrecarga independiente, las fibras se pueden hacer en un laminado por su inclusión en múltiples capas.

En la mayoría de los pacientes jóvenes con diagnóstico de labio leporino y/o paladar fisurado operado, la rehabilitación del espacio desdentado causado por la ausencia de un incisivo es una gran necesidad.

Estos pacientes generalmente presentan entre 12 y 16 ó 17 años y es en este periodo en el cual es indispensable realizar una rehabilitación provisoria que permita mejorar la estética y dar término adecuado al tratamiento odontológico. Este requisito es especialmente evidente cuando se completa el tratamiento ortodóncico y se ingresa en un periodo de espera para la rehabilitación definitiva, ya sea vía puente fijo plural o implante óseo integrado.

En la gran mayoría de estos pacientes jóvenes encontraremos que las piezas adyacentes a la zona desdentada se encuentran sanas. Por lo tanto, un tratamiento conservador en este caso sería el uso de la técnica adhesiva mediante fibras de polietileno cubiertas de plasma (Ribbond®) en conjunto con resina compuesta; y de esta forma poder confeccionar un puente adhesivo temporal utilizando el composite como póntico.

\section{CASO CLÍNICO}

La paciente M.T.F. recibió tratamiento multidisciplinario por su anomalía de labio y paladar fisurado, estuvo en tratamiento de ortodoncia de la cual fue derivada para evaluación en rehabilitación oral debido a la ausencia del incisivo lateral izquierdo, la paciente presentaba 15 años y se encontraba en periodo de contención de ortodoncia. Presentaba muy buena higiene oral, ausencia de enfermedad sistémica, facilidades para asistir a tratamiento y control, ausencia de enfermedad periodontal, dientes adyacentes a la fisura vitales, sin caries ni restauraciones, ausencia de onicofagia, por lo cual era una paciente que requería una solución estética por un período previo a una rehabilitación definitiva.

Debido a la edad de la paciente, el crecimiento y maduración de los tejidos periodontales y pulpares no se han completado, lo que contraindica una prótesis fija plural convencional.

Inicialmente a la paciente se le realizó una ficha clínica y toma de fotografías clínicas. A la segunda sesión se realizó la construcción del puente de acuerdo a los siguientes pasos:

1. Aislación relativa de la zona de trabajo intraoral.

2. Usando una piedra de diamante redonda $N^{\circ} 4$, se realizó un mínimo canal alrededor de la parte central de los dientes pilares por palatino y por proximal adyacente a la fisura.

3. Se hizo una abrasión con una piedra de forma de llama en el lado vestibular y proximal.

4. Los dientes fueron limpiados y secados.

5. El esmalte fue grabado usando ácido ortofosfórico al $37 \%$ por 30 segundos, luego se realizó un lavado por 30 segundos y se secó.

6. Se aplicó adhesivo universal.

7. Se colocó una capa de resina compuesta en el canal donde será alojado el Ribbond $\AA$ de grosor $3 \mathrm{~mm}$. Éste fué humectado con el líquido adhesivo e inmediatamente ubicado sobre el composite no polimerizado, uniendo ambos dientes en un lazo. La resina compuesta fue polimerizada junto con el Ribbond® y el adhesivo. Se agregó resina compuesta tallando directamente el diente que estaba ausente y se le colocó la capa de Ribbond® vestibular de refuerzo de $2 \mathrm{~mm}$ de grosor.

8. Terminación, se completó el fotocurado.

Se retiró la aislación relativa y se adaptó la zona gingival con resina compuesta para terminar el póntico. Posteriormente, se procedió a pulir la resina y se comprobó que la cinta no presentara zonas expuestas al medio bucal.

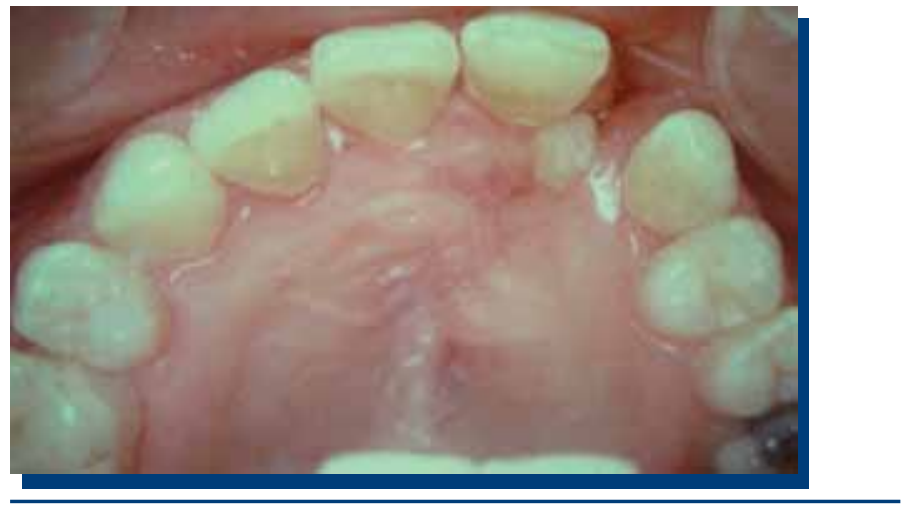

Figura 5. Zona de la fisura con la agenesia. 


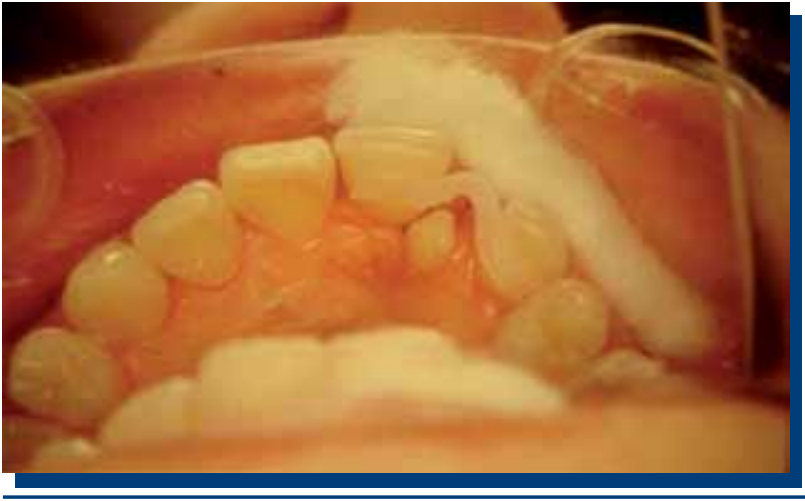

Figura 6. Inicio de la colocación del puente.

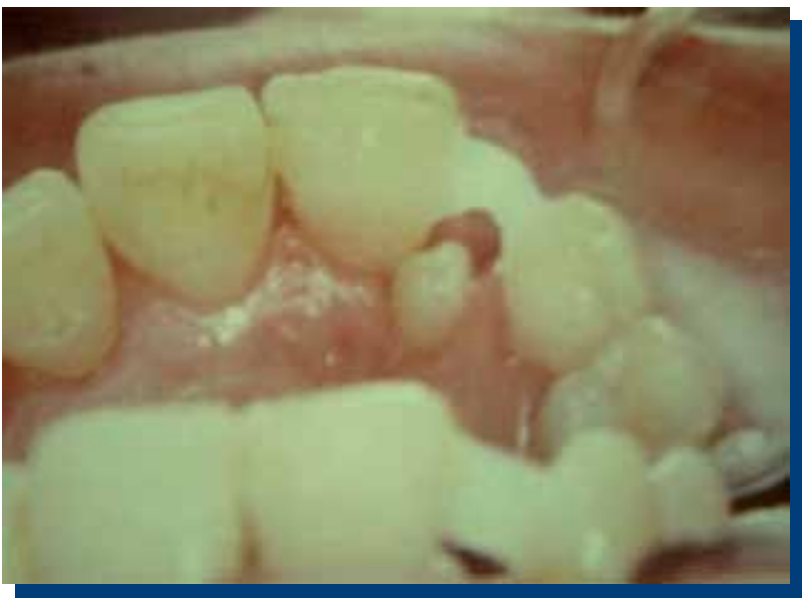

Figura 7. Ribbond® adherido.

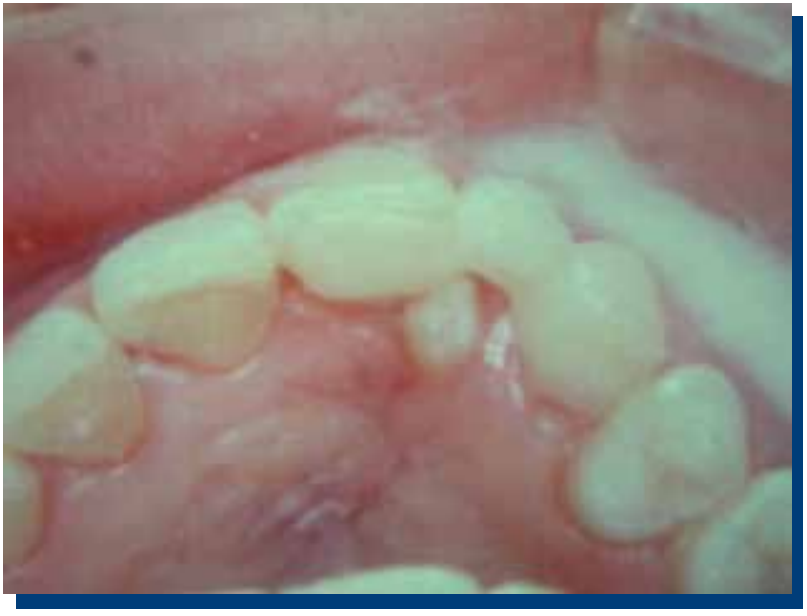

Figura 8. Tallado del diente.

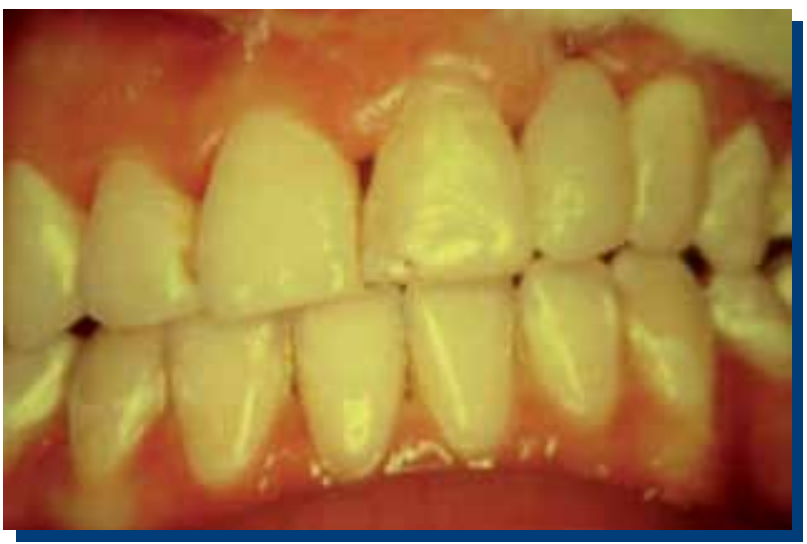

Figura 9. Finalización.

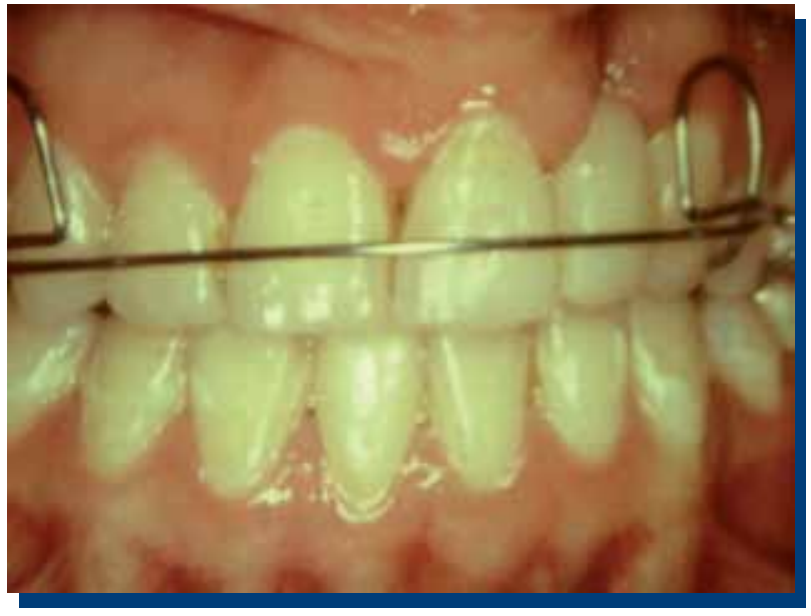

Figura 10. Con placa de contención.

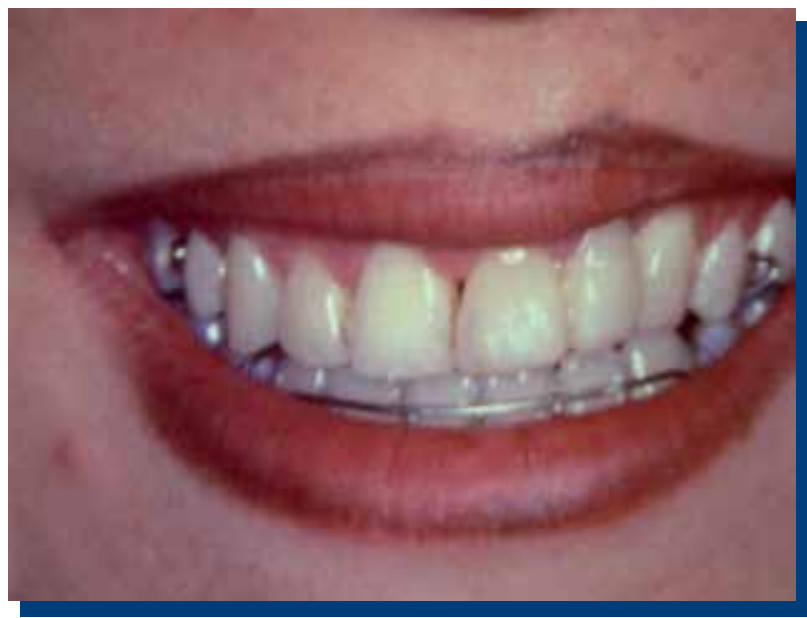

Figura 11. Puente pulido y terminado. Retiro de asa vestibular a la contención superior

\section{CONCLUSIÓN}

La gran necesidad de rehabilitaciones provisorias, que cumplan una función tanto estética como funcional, en los pacientes fisurados que se encuentran en etapa adolescente y presentan su tratamiento de ortodoncia terminado o se encuentran en periodo de contención ha llevado a la búsqueda del material más idóneo para este caso particular.

Es así como entre estos materiales restauradores encontramos como solución inmediata la utilización de la cinta Ribbond® en conjunto con resina, permitiéndonos realizar un puente adhesivo con grandes características estéticas.

Este material se destaca por sus múltiples características entre las cuales encontramos biocompatibilidad, durabilidad y estética.

Para estos pacientes jóvenes es muy importante este tipo de rehabilitación provisoria, ya que deberán esperar a terminar su crecimiento para completar la última etapa de su tratamiento el cual consisten en la rehabilitación oral de la zona de la fisura con agenesia del incisivo lateral mediante implantes o prótesis fija plural.

\section{CONFLICTOS DE INTERÉS}

Los autores declaran no tener conflictos de interés. 


\section{REFERENCIAS BIBLIOGRÁFICAS}

1. Arhun N, Arman A. Fiber-reinforced technology in multidisciplinary chairside approaches. Indian J Dent Res, 2008; 19(3): 272-277.

2. Tuloglu N, Bayrak S, Sen Tunc E. Different clinical applications of bondable reinforcement Ribbond in pediatric dentistry. EURJDENT, 2009; 3: 329-334.

3. Vitale MC, Caprioglio C, Martignone A, Marchesi U, Botticelli AR. Combined technique with polyethylene fibers and composite resins in restoration of traumatized anterior teeth. Dent Traumatol, 2004 Jun; 20(3): 172-177.

4. Uzun G, Hersek N, Tinçer T. Effect of five woven fiber reinforcements on the impact and transverse strength of a denture base resin. J Prosthet Dent, 1999 May; 81(5): 616-620.

5. DeBoer J, Vermilyea SG, Brady RE. The effect of carbon fiber orientation on the fatigue resistance and bending properties of two denture resins. J Prosthet Dent, 1984 Jan; 51(1): 119-121.

6. Berrong JM, Weed RM, Young JM. Fracture resistance of Kevlar-reinforced poly (methyl methacrylate) resin: A preliminary study. Int J Prosthodont, 1990 Jul-Aug; 3(4): 391-395.

7. Vallittu PK. Ultra-high-modulus polyethylene ribbon as reinforcement for denture polymethyl methacrylate: A short communication. Dent Mater, 1997 Nov; 13(6): 381-382.

8. Vallittu PK, Lassila VP, Lappalainen R. Transverse strength and fatigue of denture acrylic-glass fiber composite. Dent Mater, 1994 Mar; 10(2): 116-121.

9. Srinivas N, Jayanthi M, Shanthan. An innovative approach to chair side provisional replacement of an extracted anterior tooth with Fiber Reinforced Ribbon Composite for space maintenance. IJDA, 2011; 3(1): 405-408.

10. Piovesan E, Demarco F, Piva E. Fiber-reinforced fixed partial dentures: A preliminary restrospective clinical study. J Appl Oral Sci, 2006; 14(2): 100-104

11. Kargul B, Caglar E, Kabalay U. Glass fiber-reinforced composite resin as fixed space maintainers in children: 12-month clinical follow-up. J Dent Child (Chic), 2005 Sep-Dec; 72(3): 109-112.

12. Kirzioğlu Z, Ertürk MS. Success of reinforced fiber material space maintainers. $J$ Dent Child (Chic), 2004 May-Aug; 71(2): 158-162.
13. Lambert J. The natural tooth pontic; simplified. CDA Journal, 2004; 32(3): 257-260. 14. Freilich MA, Meiers JC, Duncan JP, Eckrote KA, Goldberg AJ. Clinical evaluation of fiber-reinforced fixed bridges. J Am Dent Assoc, 2002 Nov; 133(11): 1524-1534

15. Unlu N, Belli S. Three-year clinical evaluation of fiber-reinforced composite fixed partial dentures using prefabricated pontics. J Adhes Dent, 2006 Jun; 8(3): 183-188. 16. Grandini S, Goracci C, Tay FR, Grandini R, Ferrari M. Clinical evaluation of the use of fiber posts and direct resin restorations for endodontically treated teeth. Int $J$ Prosthodont, 2005 Sep-Oct; 18(5): 399-404.

17. Strassler HE, Scherer W, LoPresti J, Rudo D. Long term clinical evaluation of a woven polythylene ribbon used for tooth stabilization and splinting. $J$ Israel Orthod Soc, 1997; 7: 11-15

18. Cenci M, Rodolpho P, Pereira-Cenci T, Del Bel Cury A, Demarco F. Fixed partial dentures in an up to 8-year follow-up. JAOS, 2009: 364-371.

19. Edelhoff D, Spiekermann H, Yildirim M. Metal-free inlay-retained fixed partial dentures. Quintessence Int, 2001 Apr; 32(4): 269-281.

20. Vallittu PK, Sevelius C. Resin-bonded, glass fiber-reinforced composite fixed partial dentures: A clinical study. J Prosthet Dent, 2000 Oct; 84(4): 413-418.

21. Behr M, Rosentritt M, Handel G. Fiber-reinforced composite crowns and FPDs: A clinical report. Int J Prosthodont, 2003 May-Jun; 16(3): 239-243.

22. Song HY, Yi YJ, Cho LR, Park DY. Effects of two preparation designs and pontic distance on bending and fracture strength of fiber-reinforced composite inlay fixed partial dentures. J Prosthet Dent, 2003 Oct; 90(4): 347-353.

23. Ayna E, Celenk S. Polyethylene fiber-reinforced composite inlay fixed partial dentures: Two-year preliminary results. J Adhes Dent, 2005 Winter; 7(4): 337-342.

24. Göhring TN, Roos M. Inlay-fixed partial dentures adhesively retained and reinforced by glass fibers: Clinical and scanning electron microscopy analysis after five years. Eur J Oral Sci, 2005 Feb; 113(1): 60-69.

25. Monaco C, Ferrari M, Miceli GP, Scotti R. Clinical evaluation of fiber-reinforced composite inlay FPDs. Int J Prosthodont, 2003 May-Jun; 16(3): 319-325. 